\title{
The Exact and Approximate Solutions of Some Boundary Value Problems in Domains with Angular Points of the Boundary
}

\author{
Irina Menshova, Mikhail Kovalenko \\ Institute of Earthquake Prediction Theory and Mathematical Geophysics RAS, Moscow, Russia \\ Email: menshovairina@yandex.ru
}

How to cite this paper: Menshova, I. and Kovalenko, M. (2017) The Exact and Approximate Solutions of Some Boundary Value Problems in Domains with Angular Points of the Boundary. Journal of Applied Mathematics and Physics, 5, 39-44. http://dx.doi.org/10.4236/jamp.2017.51006

Received: November 14, 2016 Accepted: December 28, 2016 Published: January 4, 2017

\begin{abstract}
On the basis of the exact solution of biharmonic problems of elasticity theory in a half-strip one possible reason is shown of those problems that arise when an approximate or numerical approaches leading the solution of boundary value problems to infinite systems of linear algebraic equations. Construction of exact solutions of some boundary value problems for differential equations in partial derivatives is not possible without their extensions to Riemann surfaces. Moreover, each of the boundary value problem corresponds to its Riemann surface. This fact is important to consider when developing an effective approximate and numerical methods of solving boundary value problems.
\end{abstract}

\section{Keywords}

Boundary Value Problems, Exact and Approximate Solutions

\section{Introduction}

In the articles [1] [2] the theory was developed and the examples of exact solutions of biharmonic problem of elasticity theory in a half-strip and in a rectangle was first obtained (an overview of biharmonic problem for almost 200 years is given in the article [3]). These solutions are fundamentally different from all known exact solutions of boundary value problems for biharmonic equation. This is due to the finiteness of the domain and the presence of angular points of the boundary. Below there is the solution of the principal boundary value problem of elasticity theory in a half-strip. From the analysis of exact solutions the deep understanding of both mathematical and physical problem essence can be very fruitful in creating the effective numerical or approximate methods of solving variety boundary value problems in domains with angular boundary points and points of changing of the boundary conditions type. 


\section{The Solution of the Boundary Value Problem of Elasticity Theory in a Half-Strip}

Consider the solution of boundary value problem of elasticity theory for the biharmonic equation in the half-strip $\{\Pi: x \geq 0,|y| \leq 1\}$, the long sides of which are free, i.e. stresses are:

$$
\sigma_{y}(x, \pm 1)=\tau_{x y}(x, \pm 1)=0,
$$

and at the end of the half-strip the normal and tangential stresses are defined:

$$
\sigma_{x}(0, y)=\sigma(y), \tau_{x y}(0, y)=\tau(y) .
$$

There $\sigma(y), \tau(y)$ are known boundary functions.

Damped at infinity solution can be represented by separation of variables in the form of series as follows $\left(\operatorname{Re} \lambda_{k}<0\right)$ :

$$
\begin{aligned}
& \sigma_{x}(x, y)=\sum_{k=1}^{\infty} a_{k} s_{x}\left(\lambda_{k}, y\right) e^{\lambda_{k} x}+\overline{a_{k}} s_{x}\left(\overline{\lambda_{k}}, y\right) e^{\overline{\lambda_{k} x}}, \\
& \sigma_{y}(x, y)=\sum_{k=1}^{\infty} a_{k} s_{y}\left(\lambda_{k}, y\right) e^{\lambda_{k} x}+\overline{a_{k}} s_{y}\left(\overline{\lambda_{k}}, y\right) e^{\overline{\lambda_{k} x}}, \\
& \tau_{x y}(x, y)=\sum_{k=1}^{\infty} a_{k} t_{x y}\left(\lambda_{k}, y\right) e^{\lambda_{k} x}+\overline{a_{k}} t_{x y}\left(\overline{\lambda_{k}}, y\right) e^{\overline{\lambda_{k} x}} .
\end{aligned}
$$

Functions $s_{x}\left(\lambda_{k}, y\right), s_{y}\left(\lambda_{k}, y\right)$ and etc. are called the Fadle-Papkovich eigenfunctions. In the case of symmetric deformation of the half-strip they are equal:

$$
\begin{gathered}
s_{x}\left(\lambda_{k}, y\right)=(1+\mu) \lambda_{k}\left\{\left(\sin \lambda_{k}-\lambda_{k} \cos \lambda_{k}\right) \cos \lambda_{k} y-\lambda_{k} y \sin \lambda_{k} \sin \lambda_{k} y\right\}, \\
s_{y}\left(\lambda_{k}, y\right)=(1+\mu) \lambda_{k}\left\{\left(\sin \lambda_{k}+\lambda_{k} \cos \lambda_{k}\right) \cos \lambda_{k} y+\lambda_{k} y \sin \lambda_{k} \sin \lambda_{k} y\right\}, \\
t_{x y}\left(\lambda_{k}, y\right)=(1+\mu) \lambda_{k}^{2}\left\{\cos \lambda_{k} \sin \lambda_{k} y-y \sin \lambda_{k} \cos \lambda_{k} y\right\},
\end{gathered}
$$

and $t_{x y}\left(\lambda_{k}, \pm 1\right)=s_{y}\left(\lambda_{k}, \pm 1\right)=0$, therefore the boundary conditions on the longitudinal sides of the half-strip $\tau_{x y}(x, \pm 1)=\sigma_{y}(x, \pm 1)=0$ are performed automatically, $\mu$-Poisson's ratio. Numbers $\lambda_{k}$ are the set of $\left\{ \pm \lambda_{k}, \pm \bar{\lambda}_{k}\right\}_{k=1}^{\infty}=\Lambda$ all complex zeros of the entire function

$$
L(\lambda)=\lambda+\sin \lambda \cos \lambda
$$

The numbers $a_{k}, \bar{a}_{k}$ are unknown expansion coefficients.

Satisfying using expressions (2.3) that given at the end of the half-strip the normal $\sigma(y)$ and tangential $\tau(y)$ stresses, we come to the problem of determining the coefficients $a_{k}, \bar{a}_{k}$ from the boundary conditions

$$
\sigma(y)=\sum_{k=1}^{\infty} a_{k} s_{x}\left(\lambda_{k}, y\right)+\overline{a_{k}} s_{x}\left(\overline{\lambda_{k}}, y\right), \tau(y)=\sum_{k=1}^{\infty} a_{k} t_{x y}\left(\lambda_{k}, y\right)+\overline{a_{k}} t_{x y}\left(\overline{\lambda_{k}}, y\right) .
$$

Systems of Fadle-Papkovich functions do not form a basis in the classic sense. Therefore it is impossible to find the explicit expressions for the unknown coefficients of the expansions. It is the essence of biharmonic problem. However, we can construct the biorthogonal systems and functions defined on the Riemann surface of the logarithm and thereby to obtain a solution. 
The solution scheme is the following. First, we study the expansions of only one function at any one system of Fadle-Papkovich functions. Biorthogonal system of functions is constructed for it, due to we can find the expansion coefficients. It is called Lagrange expansions. Lagrange expansions are analogues of expansions in trigonometric Fourier series and play the same role in the determination of the expansion coefficients (2.6), where Fourier series engage in classical solutions of Filon and Ribiere. Then desired expansion coefficients are determined from the expansions (2.6).

Consider the system of functions $\left\{s_{x}\left(\lambda_{k}, y\right)\right\}_{k=1}^{\infty}$. Explicit expressions for the functions $X_{v}(y)$ forming the biorthogonal system to it, are determined by solving the equations

$$
\int_{-\infty}^{\infty} s_{x}(\lambda, y) X_{v}(y) d y=\frac{\lambda L(\lambda)}{\lambda^{2}-\lambda_{v}^{2}}, \quad \lambda_{v} \in \Lambda \quad(v \geq 1),
$$

where

$$
s_{x}(\lambda, y)=(1+\mu) \lambda\{(\sin \lambda-\lambda \cos \lambda) \cos \lambda y-\lambda y \sin \lambda \sin \lambda y\} .
$$

Accepting $\lambda=\lambda_{k}$ we get biorthogonality relation for the system of FadlePapkovich functions

$$
\int_{-\infty}^{\infty} s_{x}\left(\lambda_{k}, y\right) X_{v}(y) d y= \begin{cases}M_{k}=\cos ^{2} \lambda_{k} & (k=v), \\ 0 & (k \neq v) .\end{cases}
$$

The concept of biorthogonality includes the following equalities:

$$
\int_{-\infty}^{\infty} s_{x}\left(\overline{\lambda_{k}}, y\right) \overline{X_{v}(y)} d y= \begin{cases}\overline{M_{k}} & (k=v), \\ 0 & (k \neq v)\end{cases}
$$

and

$$
\int_{-\infty}^{\infty} s_{x}\left(\lambda_{k}, y\right) \overline{X_{v}(y)} d y=\int_{-\infty}^{\infty} s_{x}\left(\overline{\lambda_{k}}, y\right) X_{v}(y) d y=0 .
$$

For the complex values of parameter $\lambda$ the integrals (2.9)-(2.11) do not exist. But they can exist by appropriate deformation of the integration contour. In particular, if in (2.9) we can assume that $\operatorname{Re} \lambda_{k}>0$, the integral is taken over the contour consisting of semiaxis $x \leq 0$ and segment $|y| \leq 1$. Biorthogonal functions $X_{v}(y)$ can be represented as follows:

$$
X_{v}(y)= \begin{cases}x_{v}(y), & |y| \leq 1 \\ x_{v}^{*}(y), & |y|>1\end{cases}
$$

where

$$
x_{v}(y)=\frac{\cos \lambda_{v} y}{2(1+\mu) \lambda_{v} \sin \lambda_{v}} .
$$

It is obvious that $x_{v}(y)$ is finite part, and $x_{v}^{*}(y)$ is not finite part of functions $X_{v}(y)(v \geq 1)$. As the functions $X_{v}(y)$ are nonzero at $|y|>1$, to spread certain defined on the segment $[-1,1]$ function $f(y)$ in Lagrange series 


$$
f(y)=\sum_{k=1}^{\infty} A_{k} s_{x}\left(\lambda_{k}, y\right)+\overline{A_{k}} s_{x}\left(\overline{\lambda_{k}}, y\right),
$$

At first we should someway continue it outside the segment $[-1,1]$ on the entire real axis. In particular, when the continuations of spread functions are performed periodically with a period equal to two, we get

$$
A_{k}=\frac{f_{k}}{M_{k}}, f_{k}=\int_{-1}^{1} f(y) x_{k}(y) d y .
$$

Then

$$
f(y)=\sum_{k=1}^{\infty}\left\{f_{k} \frac{s_{x}\left(\lambda_{k}, y\right)}{M_{k}}+\overline{f_{k}} \frac{s_{x}\left(\overline{\lambda_{k}}, y\right)}{\overline{M_{k}}}\right\} .
$$

Lagrange expansion (2.16) can be considered as a generalization of expansion in a trigonometric Fourier series. Note that if numbers $\lambda_{k} \rightarrow k \pi$, then series (2.16) becomes an ordinary Fourier series for $f(y)$. By analogy with the Fourier series, the coefficients $A_{k}=f_{k} / M_{k}$ are called the Lagrange coefficients. The normalizing factor $M_{k}$ is defined as the right-hand side of expression (2.7) when $\lambda \rightarrow \lambda_{k}$. Lagrange expansions on other systems of Fadle-Papkovich functions have the same structure.

In the expansions (2.6) of two functions, in contrast to the Lagrange expansion of one function, we should find a set of coefficients $\left\{a_{k}, \bar{a}_{k}\right\}_{k=1}^{\infty}$ that will be common to these two expansions. Lacking required arbitrary is provided the existence of imaginary vectors included in the expansions (2.6). Because they do not give projections on material directions, so their expansions were called as null series. Null series should be allocated in the final expressions for the stresses and displacements in the half-strip leading them to the Lagrange expansions.

We give the final formulas for the stresses in the half-strip for those case, when at the end of the half-strip only normal self-balanced stresses are set $\sigma_{x}(0, y)=\sigma(y)$, and tangential are equal to zero:

$$
\begin{array}{r}
\sigma_{x}(x, y)=\sum_{k=1}^{\infty} 2 \operatorname{Re}\left\{\sigma_{k} \frac{s_{x}\left(\lambda_{k}, y\right)}{M_{k}} \frac{\operatorname{Im}\left(-\bar{\lambda}_{k} e^{\lambda_{k} x}\right)}{\operatorname{Im}\left(\lambda_{k}\right)}\right\} \\
\sigma_{y}(x, y)=\sum_{k=1}^{\infty} 2 \operatorname{Re}\left\{\sigma_{k} \frac{s_{y}\left(\lambda_{k}, y\right) \lambda_{k} \overline{\lambda_{k}}}{M_{k} \lambda_{k}^{2}} \frac{\operatorname{Im}\left(-\lambda_{k} e^{\lambda_{k} x}\right)}{\operatorname{Im}\left(\lambda_{k}\right)}\right\} \\
\tau_{x y}(x, y)=\sum_{k=1}^{\infty} 2 \operatorname{Re}\left\{\sigma_{k} \frac{\tau_{x y}\left(\lambda_{k}, y\right)}{\lambda_{k} M_{k}} \lambda_{k} \bar{\lambda}_{k} \frac{\operatorname{Im}\left(-e^{\lambda_{k} x}\right)}{\operatorname{Im}\left(\lambda_{k}\right)}\right\}
\end{array}
$$

Lagrange coefficient is

$$
\sigma_{k}=\int_{-1}^{1} \sigma(y) x_{k}(y) d y=\int_{-1}^{1} \sigma(y) \frac{\cos \lambda_{k} y}{2(1+\mu) \lambda_{k} \sin \lambda_{k}} d y .
$$

\section{Conclusions}

1) The solution of the boundary value problem in a half-strip is not unique. Nonuniqueness is associated with non-finite of biorthogonal functions and, as a 
consequence, the necessity of continuation the given at the end face of the halfstrip boundary functions from the segment (the end face of the half-strip) to the whole real axis. From how to carry out this continuation the problem's solution will depend. The method of a continuation is determined from physical considerations. For example, it is possible to choose an extension of boundary functions in outside the segment that at the angular point of the half-strip there will be singularity in the stresses, characteristic for the half-plane, where the part of a rectilinear boundary is rigidly clamped, and the external loads is attached on the other part. Special place is occupied by the periodic continuation of the boundary functions in which they are represented by series in systems of functions $\{\cos k \pi y\}_{k=1}^{\infty}$ or $\{\sin k \pi y\}_{k=1}^{\infty}$ (depending on the parity). First of all, the significance of these continuations is that in case the additional information, associated with continuation of the end functions (except the periodicity conditions), is not entered into the decision.

2) The construction of exact solutions is also connected with the extensions of the right half-strip to the left (even-symmetric and odd-symmetric method)with the same boundary conditions on the long sides, that on the right. However, the solution can be extended in the left half-strip and with other boundary conditions on its long sides (but with the same boundary conditions at the end of the right half-strip; i.e. in a half-strip with rigidly clamped long sides).In this case, we get another solution in the right half-strip. In particular, the stresses at the changing type's points of the boundary conditions for such extension will have a power singularity. The examples of such solutions can be found in the article [4].

3) Thus, in the exact solutions of boundary value problems the angular points of the half-strip should be considered as the intersection points of two mutually perpendicular directions, along which the continuations of the boundary functions on infinite straight line should be given to ensure the uniqueness of the solutions. In other words, in the angular points of the boundary values of the boundary functions should be defined together with all their derivatives. Consequently, it is not actual point as mathematical objects, but infinitesimal elements, that similar to the other points of the domain. It is necessary to take into account this fact in the approximate and numerical methods of solving boundary value problems for differential equations in partial derivatives in finite domains with angular boundary points and changing type's points of the boundary conditions. Since the network parameters, the choice of certain basic system functions, using of anti-smoothing procedures and etc. will inevitably affect to the behavior of infinite systems of algebraic equations.

\section{References}

[1] Kovalenko, M.D. and Shulyakovskaya, T.D. (2011) Expansion in Fadle-Papkovich Functions in a Strip. Theory Foundations. Mechanics of Solids, 46, 721-738. https://doi.org/10.3103/S0025654411050074

[2] Kovalenko, M.D., Men'shova, I.V. and Shulyakovskaya, T.D. (2013) Expansions in Fadle-Papkovich Functions: Examples of Solutions in a Half-Strip. Mechanics of Solids, 48, 584-602. https://doi.org/10.3103/S0025654413050154 
[3] Meleshko, V.V. (2003) Selected Topics in the History of Two-Dimensional Biharmonic Problem. ASME Report No AMR 341/Appl. Mech. Rev., 1, 33-85. https://doi.org/10.1115/1.1521166

[4] Kovalenko, M.D. and Shibirin, S.V. (1997) A Junction of Two Semistrips. Mechanics of Solids, 32, 45-51.

Submit or recommend next manuscript to SCIRP and we will provide best service for you:

Accepting pre-submission inquiries through Email, Facebook, LinkedIn, Twitter, etc. A wide selection of journals (inclusive of 9 subjects, more than 200 journals)

Providing 24-hour high-quality service

User-friendly online submission system

Fair and swift peer-review system

Efficient typesetting and proofreading procedure

Display of the result of downloads and visits, as well as the number of cited articles Maximum dissemination of your research work

Submit your manuscript at: http://papersubmission.scirp.org/

Or contact jamp@scirp.org 